\title{
Designing Smart Textiles Prints with Interactive Capability
}

\section{Prof. Hoda Abdel Rahman Mohamed El-Hadi ${ }^{1}$,Prof. Sherif Hassan Abdel Salam ${ }^{2}$ Eng. Kholoud Hassan Mohamed Mahmoud ${ }^{3}$}

\author{
1) Emeritus Professor, Department of Textile Printing, Dyeing and Finishing and Former Head of the Department, Faculty of Applied Arts, \\ Helwan University, Egypt \\ 2) Professor of Printing Technology, Department of Textile Printing, Dyeing and Finishing, Vice Dean for Education and Student Affairs and \\ Former Head of the Department, Faculty of Applied Arts, Helwan University, Egypt \\ 3) Freelance Engineer and $\mathrm{PhD}$ researcher in Department of textile printing, dyeing and finishing, Faculty of Applied Arts, Helwan Universi- \\ ty, Egypt.
}

\section{KEY WORDS:}

Smart Textiles -Thermochromism-Photochromism- Interactive Printed Textile Pattern -Designing Interactive (dynamic) Printed Patterns for Textiles .

\begin{abstract}
:
The present research deals with the study of Smart Textiles Prints with interactive ability (dynamic), and determining their patterns, and how the designer of interactive textile prints in this case and through a set of different approaches and design methods that are one of the most prominent forms of future design of determining the appropriate design steps according to functional and aesthetic purpose For each printing design, which is linked to the changing nature of the printing works of art that are interactive, and through research study of a range of previous experiences in the same field and the study of the views of a group of specialists and researchers have been reached A number of important variables and factors that have a direct impact on the design process and on the determination of the approach followed, the current research also deals with the study of modern technological and printing methods and the most important materials used to reach the implementation of interactive textile printings.
\end{abstract}




\section{INTRODUCTION:}

With the continuous advancement of mankind and with the advent of the "knowledge age", technological tools and materials have merged with the different materials which are present in the surrounding environment. One of those materials is textiles, The textile materials have become an area that attracts the attention of specialists and scientists who are interested in everything related to the term "smart", and this has changed the future of traditional textiles to become "smart textiles" based on the integration of electronic science, Textile and textile science products, and all associated coloring materials or processing materials, etc., and the various smart textile products are now carrying multiple functional values that change the culture of consumer or user towards those smart textile products, (1) which includes interactive (dynamic) textiles prints, and also change the form of interrelationships between them to become more interactive, in addition to that these developments put the designer or artist who is implementing and planning this type of modern textile arts in front of a set of variables and new factors that affect his designs and his choices to implement an idea, Therefore, to illustrate the process of designing interactive smart textile printing as a form of future design and one of the contemporary artistic trends, and to identify the design methodology used in this design methodology, and the factors and variables involved in this process, we discuss at the beginning of this research The definition of "Smart Textiles" in general and "Interactive Printed Textile Patterns" in particular, and also discuss the most important techniques and technological methods used in the creation and design of these interactive textile prints A special aspect, including the interpretation of the types of interactive prints (Thermochromism - Photochromism, etc.), and the various materials used in this.

\section{Smart Textiles}

\subsection{Definition}

The term "smart" is used these days to describe many forms of raw materials and textiles that have a responsive characteristic to express the abilities of those textiles. In fact, textiles cannot think smartly, but they are used to express "A certain responses to specific inputs or data through the addition of A Certain Response to A Given Input",(2) So one of the most important features of products or systems (including smart textiles) consisting of smart materials is the ability to take variable and acceptable reactions in line with their functionality, "knowing that these catalysts can arise within the inner environment of Smart textiles or outer of it (in the surrounding environmemnt)(3), from that we can define Smart Textiles generally as raw materials that interact with the surrounding environment in different ways and shapes, and also as textiles that adapt and respond to that external environment.(4)

\section{Smart Textiles classified by their Interactive Printed Pattern}

\section{1. Reversible Dynamic Pattern:}

It is a printed fabric that reacts to the effects in the surrounding environment and can return to its first form, in this case there is always a starting point and a variable number of responses to that prints.(5) For example, in Fig. (1) The colors of the design appear with the effect of UV light and with the dismiss of the effect the colors return to their original position of gray or transparent.
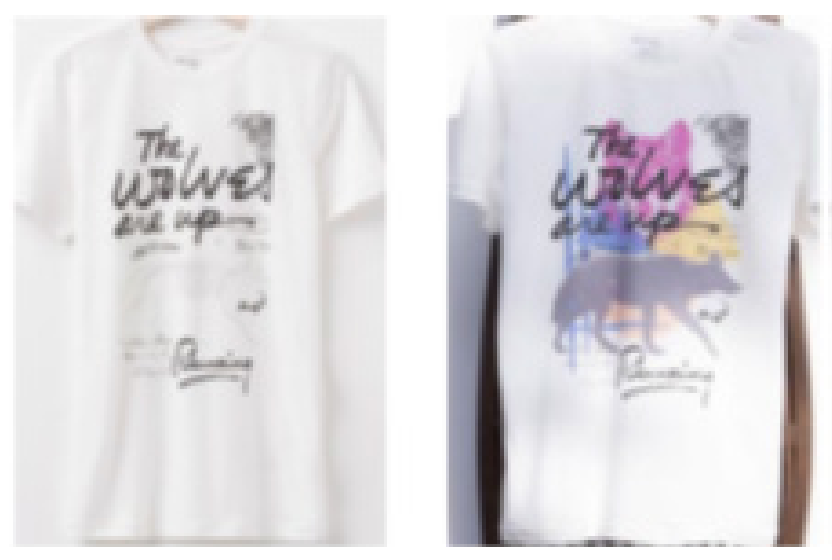

Figure (1) shows the form of interactive textile prints (Photochromic prints) before and after the effect (UV light). (Left to right)(6)

\section{2. Irreversible Dynamic Pattern:}

It is a textile pattern that changes at the time of use and does not return to its first position, and over time the extent of change increases and the accumulation of the changes made with each other.(5) For example, in Fig. (2)Textile Table Cloth which is designed according to the non-chemical combustion technique, resulting in color changes and changes in the structural composition of the textile material, as a result of different burning patterns. 


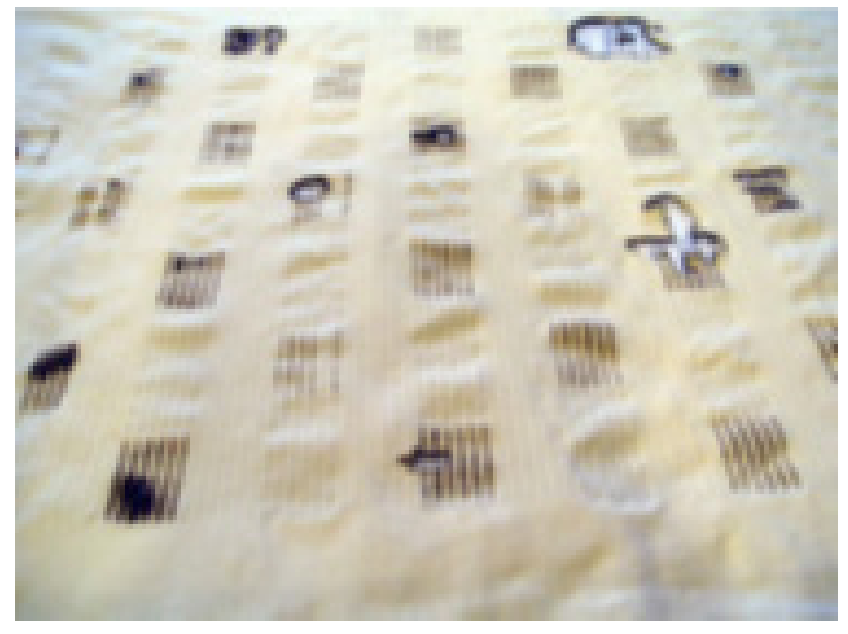

Figure (2) Interactive textile work entitled "The burning tablecloth", illustrating the effects of non-reflective gravure printing.(7)

This classification is the most generalin the field of Smart Textiles printings, taking into account that there are several other factors affecting the final result of the smart printedtextile such as the multiplicity of materials and techniques that are used. "Reversible and
Non- Reversible" Textile printed patternsare two patterns which are used in"describing emerging changes in the forms and responses of Smart Printed Textiles which make them interactive (dynamic)", from this point, The color changes occurring in the printings can be identified and controlled in the so-called "A reported pattern", which means "the interaction of printings with specific effects (whether digital or electronic). For example, to make any kind ofchange, we need a tool to press a specific area on the printed fabric, which in turn is affected and different forms of changes occur. This pattern can be controlled and can be switched on or off depending on the spatial variables and the surrounding conditions". In the other case in "A direct pattern" it is more monotonous and simpler, but it also responds according to the user's actions. In a direct pattern, change occurs according to a specific pre-planned design, and in real time, but the outcomes are unknown because they depending on the user interaction and his ability to relies the interactive printed work, in a direct pattern change occurs inthe same time and in a closer form of the human being interacting with it and with its responses.(8)

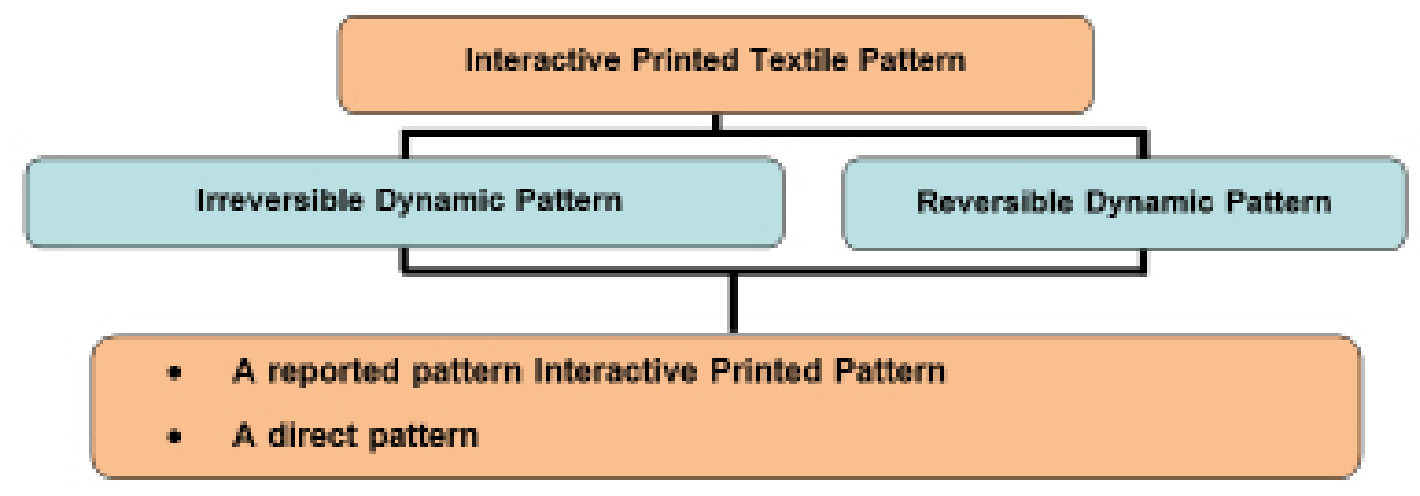

Schematic diagram Figure (3) Shows the classification of Smart Interactive Printed Textilesand their patterns

\section{Designing Interactive (dynamic) Printed Pat- terns for Smart Textiles}

\section{1. Design methodology:}

The field of designing Smart Textile in the twentieth century is one of many different areas of design in which many modern methods and design approaches have emerged. This was accompanied by the emergence of a set of concepts and terminologies related to the smart textiles design process, which would provide several interpretations of the term "Design Methodology"(9), which itself emerged as a theory, needs to be studied and examined starting in the mid-1960s, and with time and since the early nineties, "Design Methodology" has evolved considerably and many researchers have adopted many different methods to reach their goal, and the number of conferences and workshops that follow different design approaches has grown up and become a subject of research for many specialists and researchers.(10)In a statement by the Danish government on "Interactive Textiles Design" in 1997, it explains the aesthetic aspect of the design of those textiles, citing researcher "Thomas Dickson," which explained that "design is a process of expressing the process of creativity in which the physical properties of a product are transformed as well asits Aestheticproperties, design is a process that combines the nature of the product itself with the process of its creation". The researchers also explained in the same field that the design process refers to "EngineeringDesign" which intended to "the use and application of scientific principles, technical information, and perception in the creation of a body of an Automatic construction To perform specific functions efficiently and economically", "Design" also refers to "innova- 
tion and product development processes,"Researchers "Dan Whitney and Chris Magee" of the Massachusetts Institute of Technology's Product Development and Innovation Center define "design" as "reworking or reshaping customer requirements and technological possibilities in the form of integrated products". All of those previous definitions refers to that the process of "designing Smart, interactive textiles" has many interpretations and meanings that require a broader and more comprehensive framework that describes "design" as "the imaginary transition from current realities to future possibilities"(9).

This leads us to the conclusion that when we start planning the design of an interactive textile artwork we find that there are many approaches and methods that can be applied as a result of the "nature of the changing artwork" (Dynamic Pattern), and the research process in the framework of "the development of that plan" constructively A systematic process of a set of "data" which in turn necessitates a "special methodological model commensurate with the nature of the work", Figure (4).As "Kroes" stated in 2002, "methodological approaches, especially in the "interactive design"process, are a set of "processing".(11) We can say that when studying the "Design Methodology" of an interactive artwork, Several key areas, as shown in the diagram, are included as shown in Schematic diagram Figure (2), most notably:

- Design Motivations.

- Aesthetic outcomes and Innovative.

- Technologically Led Practices.(10)

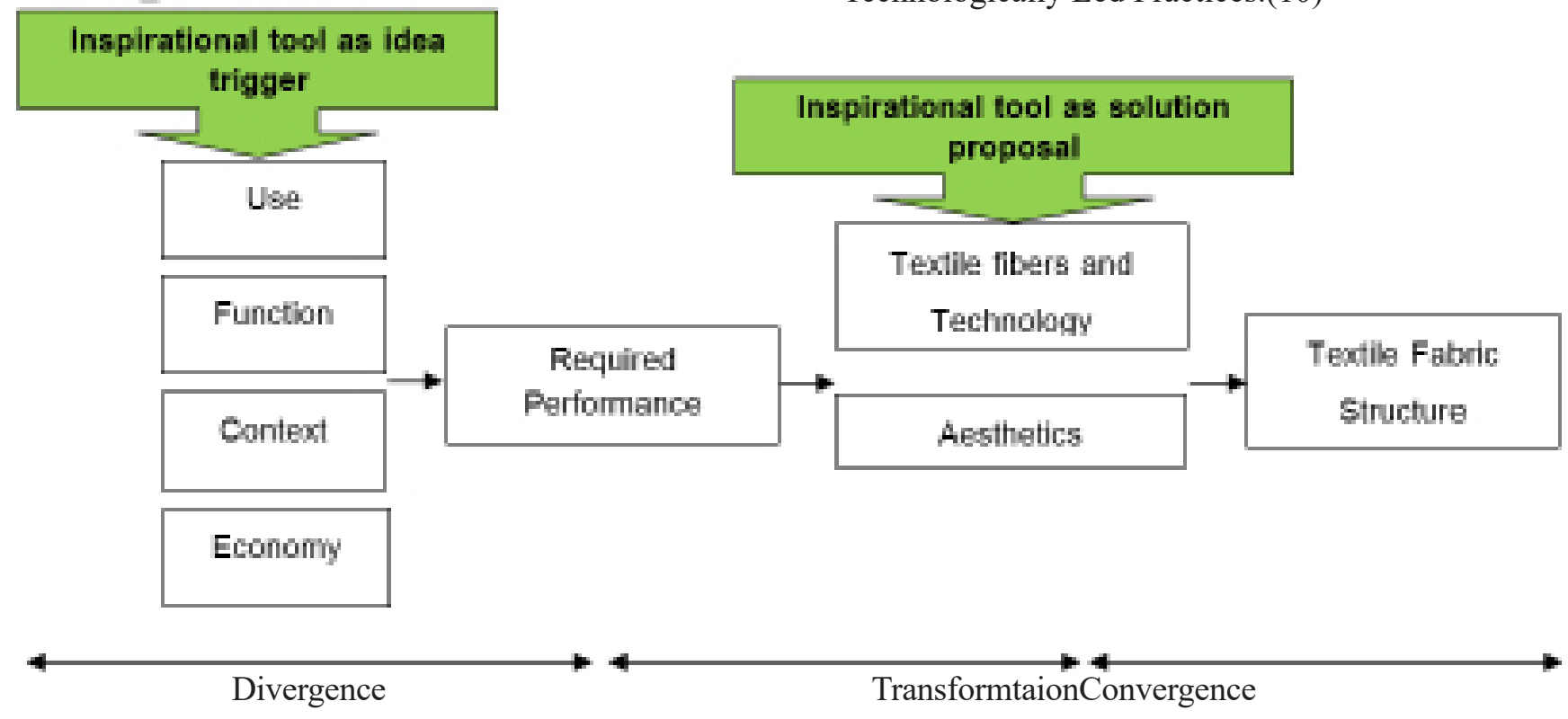

Schematic diagram Figure (4) Is illustrating the main elements of the "design methodology" for Smart Textiles in general, which includes Interactive Printed Textiles(2)

3.2. The most important variables and factors affecting the design of Interactive Printed Textiles (dynamic):

When working on the design of interactive smart printed textiles or when using modern printing materials, we cannot apply these traditional methods which used in the past except for some key steps, which also varying depending on the nature of the printed work,(10) Among the leading researchers in the field of "identification the methodology for the design of smart textiles printing with interactive ability, especially "Reversible interactive prints" researcher "Linda Robin", whose developed a system for describing interactive prints consisting of "symbols (A B A)", which symbolizes that the design changes from one static imagery (A) to another static imagery (B) and then returns to its original state (A). Linda adds that the dynamic design hasthe potential to change into one or more possible expressiondifferent from the original design of the design."(10) From that The researcher "Linda Robin" developed a set of'Dynamic Design Variables" Table Figure (5), which can be used by the designer of smart and interactive (dynamic) printed textiles as follows:(5) 
The most important variables and factors affecting the design of Interactive (dynamic) Textiles

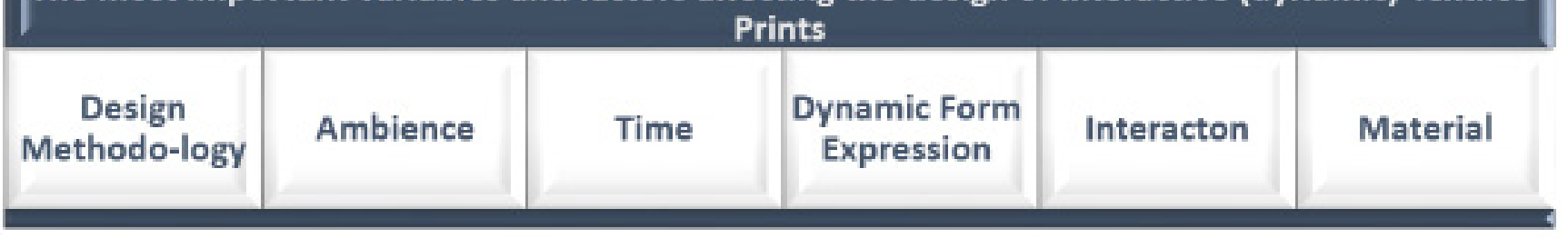

Table (5) designed by the researcher

As a result of what we have reached and the nature of the smart printed textiles, which is characterized by its interactivity (dynamic), a set of concepts and technical terms related to the design process and the stage of use have emerged, which in turn are also variables affecting the design process because of its relationship to determine the form of response and its reaction that is considered the result of an impact or to the user, Most notably:

- Degree of activationDOA: associated with the number of dynamic elements that change as a result of an effect, also defined by the type of reaction, for example the degree of burning printed on the tablecloths shown in Figure (2) above and designed so that each text message or incoming call has the effect of running a single burning operation, In this example, a user can run a certain number of elements, compared to the number of variables that have been designed for the smart printed textile.

- DOI- Degree of intensity:It is related to the amount of changes in the interactive textile material, which is also related to the reaction itself. For example, to measure the extent of color change or the change in the texture of the fabrics, as a result of an effect such as heat, light or motion. For example, the color change in the dancers' customs and wall hangings As a result of the movement of the dancers, when they stop colors gradually return to the first position, and whenever they maintain their position and the color retained its degree associated with the duration of their stop. (12) - SOA - Spot of activation: Operating points determine which variable factors will begin to operate and be affected, as well as depending on the type of reaction, for example in the case of thermal printing reaction fabric is affected in the specific printed places where the user puts a hand.

- ROA - Rhythm of activation:Defined as the relationship between dynamic elements and their interaction-catalytic, for example, the printed operating points on the table cover respond directly to the signals transmitted by the mobile phone to start burning and in another example the color change in the printed textiles wall hanging which is attached to the movement of dancers without any delay At the time of that response, but in another type or interactive printed textile Touching Loops are programmed to start responding after 10 seconds of touch, and the user can not influence or change the operating mode of these models mentioned above.
- IROA - Inherent rhythm of activation:This type is associated with rhythmic changes that are related to the spatial space or the surrounding area that stimulates the dynamic elements to start operating, which is related to the reaction process, for example, (8) one of the reactive textile printing models, which is a window cover (curtain) its printed and designed to be influenced and respond to external environmental influences such as lighting, temperature change, and electricity.(12)

These factors have been developed based on a set of experimental and applied studies of a variety of different interactive printed designs and using a range of modern materials (Photochromism, Thermochromism, etc., and different printing methods which will be discussed in detail in the next part of this study) - to reach the most important variables Which in itself serves as a plan and as a method of work for the designer of interactive printing textiles, the researcher "Linda" explained that these factors were determined as a result of the "new nature of the printed artwork" (interactive), which differs from traditional prints, She also explained that modern digital systems have an important role to play in determining the different forms of printing design and that by using these technological methods, the aesthetic and artistic aspects of the printed design can be controlled, and The interactive textile printing designer must understand the many different materials that used in the printing artwork, So that he would be able to control it as much as possible and determine the effect that will be used in the work, whether external or internal stimulus in the construction of the work itself. (10)

As a result of what we have reached and the nature of the smart printed textiles, which is characterized by its interactivity (dynamic), a set of concepts and technical terms related to the design process and the stage of use have emerged, which in turn are also variables affecting the design process because of its relationship to determine the form of response and its reaction that is considered the result of an impact or to the user, Most notably:

- Degree of activationDOA: associated with the number of dynamic elements that change as a result of an effect, also defined by the type of reaction, for example the degree of burning printed on the tablecloths shown in Figure (2) above and designed so that each text message or incoming call has the effect of running a single burning operation, In this example, a user 
can run a certain number of elements, compared to the number of variables that have been designed for the smart printed textile.

- DOI- Degree of intensity:It is related to the amount of changes in the interactive textile material, which is also related to the reaction itself. For example, to measure the extent of color change or the change in the texture of the fabrics, as a result of an effect such as heat, light or motion. For example, the color change in the dancers' customs and wall hangings As a result of the movement of the dancers, when they stop colors gradually return to the first position, and whenever they maintain their position and the color retained its degree associated with the duration of their stop. (12) - SOA - Spot of activation: Operating points determine which variable factors will begin to operate and be affected, as well as depending on the type of reaction, for example in the case of thermal printing reaction fabric is affected in the specific printed places where the user puts a hand.

- ROA - Rhythm of activation:Defined as the relationship between dynamic elements and their interaction-catalytic, for example, the printed operating points on the table cover respond directly to the signals transmitted by the mobile phone to start burning and in another example the color change in the printed textiles wall hanging which is attached to the movement of dancers without any delay At the time of that response, but in another type or interactive printed textile Touching Loops are programmed to start responding after 10 seconds of touch, and the user can not influence or change the operating mode of these models mentioned above.

- IROA - Inherent rhythm of activation:This type is associated with rhythmic changes that are related to the spatial space or the surrounding area that stimulates the dynamic elements to start operating, which is related to the reaction process, for example,(8) one of the reactive textile printing models, which is a window cover (curtain) its printed and designed to be influenced and respond to external environmental influences such as lighting, temperature change, and electricity.(12)

These factors have been developed based on a set of experimental and applied studies of a variety of different interactive printed designs and using a range of modern materials (Photochromism, Thermochromism, etc., and different printing methods which will be discussed in detail in the next part of this study) - to reach the most important variables Which in itself serves as a plan and as a method of work for the designer of interactive printing textiles, the researcher "Linda" explained that these factors were determined as a result of the "new nature of the printed artwork" (interactive), which differs from traditional prints, She also explained that modern digital systems have an important role to play in determining the different forms of printing design and that by using these technological methods, the aesthetic and artistic aspects of the printed design can be controlled, and The interactive textile printing designer must understand the many different materials that used in the printing artwork, So that he would be able to control it as much as possible and determine the effect that will be used in the work, whether external or internal stimulus in the construction of the work itself. (10)

4. The most important modern materials and methods used in the Applied field of Interactive Printed Textiles 4.1. Thermal Materials, includes:

\subsubsection{ThermoelectricMaterials:}

It consists of the merging of many materials and their functional integration such as inorganic semiconductors, conductive polymers, and various materials and fibers with electrothermal properties. The addition of these electrothermal smart fabrics can be combined with other artistic techniques ranging from covering or printing, and even to direct drawing, which will give inspiration to specialists to create intelligent, interactive textiles in the future. The Thermo Electric Generator (TEG) is an electrothermal system typically consists of a combination of two p-type and n-type electrothermal components, respectively connected to conductors, when a temperature change occurs between the two materials, through which the charge travels from The hot area to the cold zone, and the thermal energy is converted into an electric charge. (13)In the following are the main printing materials used to achieve this:

4.1.1.1. Bi \& Sb"Bi and Sb Containing Printing Inks: Bismuth or materials of Bismuth Telluride are the most distinctive electrothermal materials for generating electricity at temperatures below $200{ }^{\circ} \mathrm{C}$.(14) Commercially known as "TE" Fig. (6) and (7) are the most widely used and widely available as an electrothermal materials, they are used in various industrial fields related to the principle of the employment of thermal energy.

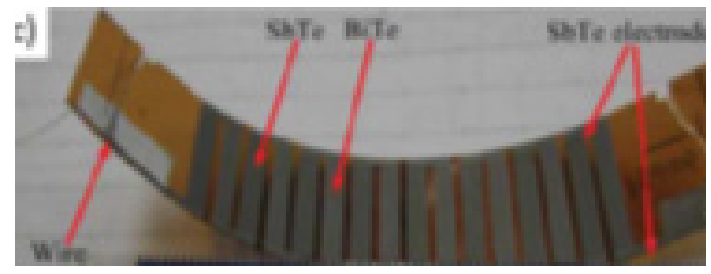

Figure (6) Bismuth Article

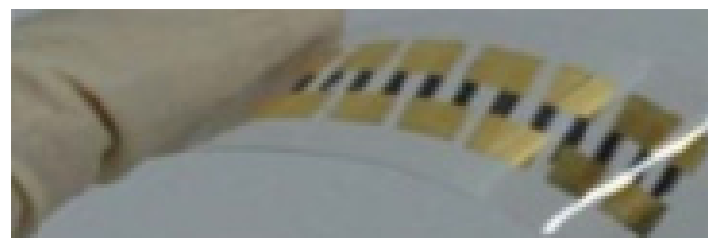

Figure(7) Another form of Bismuth (15)

\subsubsection{Nickel Printing Inks:}

Used in the manufacture of circuit boards (PCB) and electronics, nickel is one of the most important conductive printing materials. 


\subsubsection{Conducting Polymers:}

Conductors are polymeric materials with high resistance to chemical, mechanical and electrical factors, so since the beginning of their use in the manufacture of sensor parts, polymeric conductors have entered in various fields of application such as optoelectronics manufacturing and printed electronics, as well as supercapacitors and microactuators . All of these materials have important and distinctive characteristics such as solution processibility, lightweight, flexibility, optical properties, as well as electrical properties, which makes them extremely valuable and a key role in the formation and design of smart textiles in general and specifically interactive textile prints. (14)

\subsection{Chromic Materials:}

Materials that have the ability to change color due to external factors and influences, and these materials are widely used in the field of fashion to create interactive printing designs with variable colors. Depending on the nature of the effect, these substances are classified as follows:

\subsubsection{Photochromic:}

It is a material that changes color when exposed to UV-light. This effect is used in several areas including toys and plastic products, and is also characterized by its ability to return to the first color (a reversible color change), currently,appeared new pigments that can change from white color to many range of colors such as bambi or blue or turquoise or magenta red or green, and this feature is also available in threads from one color to another and not from white as in dyes or pigments, and the number of colors is limited.

\subsubsection{Thermochromic:}

For example, the colors of Thermochromic pigment TC, which change color from one degree to another depending on the temperature change, it was used in many areas such as textiles, toys, kitchen equipment and other different fields. These colors have a general characteristic, such as the ability of the color to return to the first color (basic) (a reversible change of color), and the temperatures affecting this type of coloring between $8{ }^{\circ} \mathrm{C}, 15^{\circ} \mathrm{C}, 27^{\circ} \mathrm{C}$ etc. AQ Variotherm is a concentrated type of TC pigment that works by soluble in water and can also be dissolved and mixed with traditional textile printing pigments, each with a different degree of light and washing stability (on cold $40{ }^{\circ} \mathrm{C}$ ).

\subsubsection{Electrochromic:}

For example,ELElectroluminescencematerials, the term "photoelectric" refers to the scientific phenomenon associated with the combination of light and electricity technologies, which are used for safety signals in vehicles, aircraft, billboards and as backlights in thin LCD TVs, lighting lamps, etc. These colors are available in the form of film sheets or wires can be cut in different lengths and sizes. To illustrate, photoelectric dyes result from the addition of phosphorus on conductive prints or on any cell as a conductive core (whether film sheets or wires), as well as an electrode and a protective encapsulation container. EL can obtain light by operating a power source, and comparing to other light sources it is a low-power consumption technology that does not produce overheating, but requires a high-voltage $\mathrm{AC}$ transformer.(5)

\subsubsection{Mechanochromic/Piezorochromic:}

The materials that able to change there color due to a pressure on it. There are various forms of pressure, simple or complex, such as tensile pressure. Another term used as an alternative to piezochromic is mechanochromic. The piezochromic colors are less common and less used in different interactive printing experiments, especially compared to photochromic colorants.

It is used in this type of interactive printing materials that are affected by pressure, polymers and inorganic materials, such as Cyano oligo (a poly-phenylene vinylenes (p-phenylene vinylenes), which has the properties of flexible plastic materials, that are affected by heat. Recently, a mixture of polymers has been developed to create different interactive dyes, for example a mixture of polymers containing cholesteryl derivatives to create and design smart dyes that can respond to the least possible compressive effect and show a response in color changing from red to green.

\subsubsection{Solvate chromic:}

They are substances that change color as a result of being affected by a solvent. It is recognized that some chemical compounds are sensitive to a solvent (gas or liquid), the chemical nature of solvents requires the presence of other environmental factors surrounding the substance in order to operate at their usual efficien$\mathrm{cy}$, and to determine the percentages of the appropriate concentration to affect the chromium group in sensitive printing dyes.

\subsubsection{Chemochromic:}

Materials that change color due to chemical changes in the surrounding environment. This technique is used, for example, in the design of double glazed windows affected by hydrogen gas, also follow this categorythe chemical-sensitive color groups divided as follows:

- Halochromic: they change color due to the change in $\mathrm{pH}$ in the surrounding environment.

- Ionochromic: Color changes due to the presence of an ionic effect 


\subsubsection{Biochromic:}

These colorants are affected by the presence of germs or microorganisms and ithas been working on this type of material and developed with the aim of identifying and recording the presence of organisms, which is used in various areas of protection from food poisoning or the prevention of biological warfare as sensitive and interactive sensors.

\subsubsection{Hydrochromic:}

They are substances that change color as a result of contact with water or if they are in damp places. The color molecule in this group also follows the category of sensitive colorants affected by different solvents. (16)

\subsection{Illuminates Materials, includes:}

\subsubsection{ElectroluminescentMaterials:}

Many of the bright, smart textiles are used in a variety of applications, including in the garment industry, interior design and advertising, but they are more practical and suitable for the protective and sports garments industry to provide a degree of security for the user (because he becomes illuminated) and becomes more visible. At present, this phenomenon depends on the printing of light emitting devices (LED). There is also ACPEL material, it is an acronym for (alternating current powder electroluminescence), which can be printed using silk screens, as shown in Figure (8), where (A) SEM (Scanning electron microscopy) forms of ACPEL (B) Photos of the fabric printed with the ACPEL as it is lighting.
A

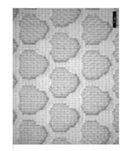

B

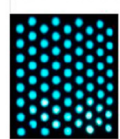

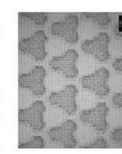

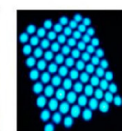

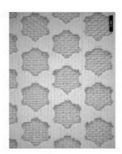

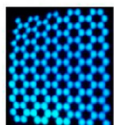

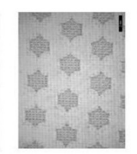

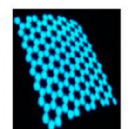

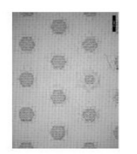

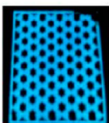

Figure (8) illustrates the effect after printing the ACPEL. (17)

\subsubsection{Fluorescent Materials:}

For example, Photoluminescent colorants, which have the ability to absorb natural or artificial light and reemit a constant glow in the dark and in a quick time, the dye continues to glow until energy is depleted, and can be recharged repeatedly by re-exposure to light. (18)

\subsubsection{PhosphorescentMaterials:}

It is the most commonly used phosphorant colorant, which absorb light and re-emit a pale blue light in a slow time, this colorants are the most widely using which are made from a mixture of zinc sulfide and copper, while green phosphoric dyes contain Sodium fluoresce, and red color of contain Rhoda mineral.(19)

\subsection{Conductive Printing Materials, includes: 4.4.1.Inherently conductive polymers:}

These polymers are capable of both sensing and actuation, such as (ICPs)polymers which are widely known,they include (polyacetylene, polypyrrole, and polyaniline). "PPy" (polypyrrole polymer)is the most appropriate for use due to its strong mechanical properties and high flexibility, and stability against weather and electrical factors. One of the most important characteristics of polymers as a sensor and a good conductor is that they maintain the nature of the elastic fabric, but their disadvantages are the loss of responsiveness or the ability to feel over time.(20)

\subsubsection{PiezoelectricMaterials:}

Piezoelectricity refers to electricity produced by a pressure effect, which makes these materials very good sensors as well. The electrically conductive compressive materials are divided into two main groups:

- Crystals of ( $\mathrm{SiO})$

- polymers.

Also a form of electrically conductive materials that used in the field of smart textiles,rolls of thin LCD displays, and conductive filmsthat have the ability to withstand the pressure on them, and the liquid color containing liquid crystals paint. (21)

\subsubsection{Conductive Printing inks:}

Different textiles can be printed with a layer of conductive inks fig.(9), using screen-printed method, which gives them the ability to conduct in those specified printed places. Its also possible to add different conducting materials to traditional printing pigments such as (Copper, silver, nickel, gold), these conductive printing places are employed at a later stage to become operating points for integrated electrical and electronic circuits and systems.

\subsection{Chemical Treatments \& Interactive coloring, includes:}

\subsubsection{Conductive Coating:}

Traditional fabric can also be transformed into intelligent conductive materials through the use of electrically conductive lining. This lining is suitable for the use of many types of fibers and textiles. Such as:

- Non-electroplating process for fabric.

- The process of lining the fabric using an electrically conductive polymer(22) 


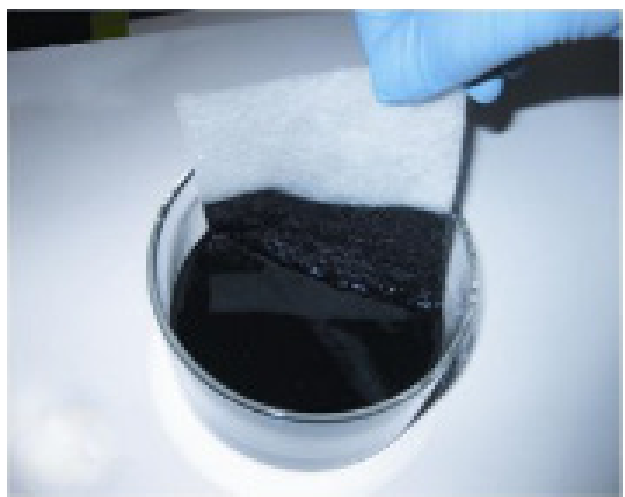

Figure (9) Conductive inks.(20)

\subsubsection{Recoloring with Fabrics Pens:}

Fabric pencils are a transparent dye pencils with a headin many sizes. They are specially made for use on fabric. They are easy to use. Suitable for linear work with fine details such as Fig. (10), they are non-toxic and are sufficient to dye large areas of fabric several times if necessary to give a dark tint of color, it can be diluted with water to obtain a light tint of dye, fixed with a hot iron on the reverse side of the fabric, then it can be washed, and two colors can be applied on top of each other to form a new color. Make a gradient of two degrees of dye while applying it on the canvas by itself, by pumping the other colored head up and down. This type of dye does not appear visible on fabrics with dark colors, and some types can be refilled with the dye, or change its head.

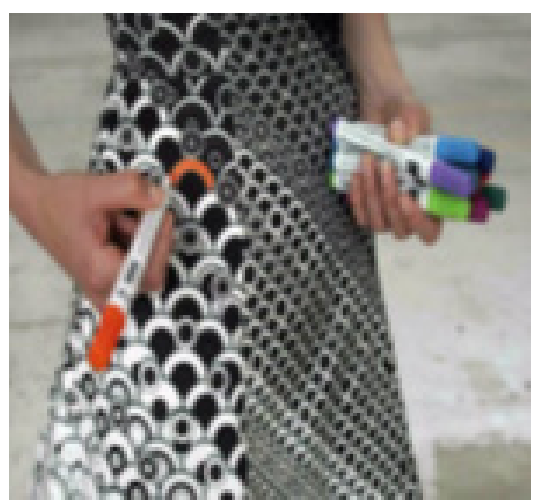

Figure (10) shows the user's ability to directly color the printed design according to his choice and his own vision.(23)

\section{Fields of application}

Thermochromic and Photochromic dyes have been widely used in textile applications (figure 11), specifically, they have brought novelty into fashion industry. However, this is not the only field in which those pigments are being used. Variety of non-textile applications and product manufacturers are also consumers of thermochromic pigments. Thermometers,temperature indicators and body monitoring devices can be count- ed among the non-textile applications. During the recent years, smart textile section has opened new ways to functionalize thermochromism. Specially designed T-shirts capable of showing wearer's body temperature is an example of such innovations. (24)Moving from gadget based approach to integrated smart fabrics, hand in hand with the fashion industry creates substantial opportunities at the global level. According to Global Market Insightsthe market (figure 12) for Smart and Interactive Textiles will surpass $\$ 6.5$ billion by 2024, where Europe will account for more than a fifth of the globalsmart and interactive textiles market. It's expected strong and fastgrowth in the regions of North America, Europe and Asia Pacific.The tech-savvy population of China, Japan, and South Korea will propel the Asia Pacific smart and interactive textiles market growth, moreover it is forecasted that Military projects in the U.S., China and South Korea can have the effect of boosting the growth in these regions. Another analyst (Ameri Research Inc.) predicted an even higher growth (x13) of the global market for smart textiles, raising from USD 674,8 Million in 2015 to reach above USD 8980 million by 2024 as we can observe in the following figure in which it can be observed the growth by regions. This change of speed in the growth of this market, as compared to the recent years, is mainly motivated by the significant investments of large companies and their quick release of commercial products.

The Smart Fabric sector has emerged during the last years thanks to the sensing devices that can be integrated into smart materials and their connectivity options giving them more and more IoT characteristics. These E-Textiles provide advanced functions thanks to their capacity to sense and react to the environment and they are taking advantage of the miniaturization of electronics in combination with the decreasing costs for the manufacturing of these high-tech materials. This evolution allows them to be used in very diverse scenarios ranging from individuals and occupational groups with the need of fabricbased services to military professionals for safety and protection. Healthcare and the need for global tele monitoring programs will be one of the areas that will drive the growth for smart interactive fabrics in the coming years. This will be speed up by the needs from doctors to have better sensors integrated in textiles in order to have better prevention systems.

According to these market trends, we predict that this growth can be organized in three different phases 
aligned with the evolution of the technology and its adoption by the market as it is detailed in the following figure. Obviously, all use-cases (all needs) do not have to go through all these phases, in many cases, something attached to the fabrics will be sufficient. Early adopters will be people that have a specific usage of these textiles like military people or athletes. The evolution will be similar to the one we observed in the mobile industry, evolving from big and heavy devices in the seventies to smart devices like the iPhoneas we have today. In this evolution, new value chains will be created in order to allow moving from some prototypes as we have today to a massive production.From business case perspective, we can refer Smart Fabrics to multi sided market with brands, customers, manufacturers, retailers coming together to create an economy to make this cost effective.

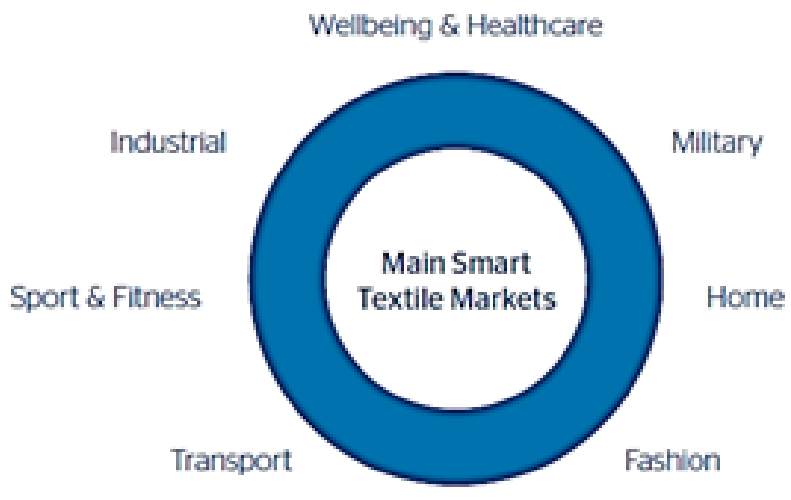

Figure (11): Main Markets for Smart Fabrics

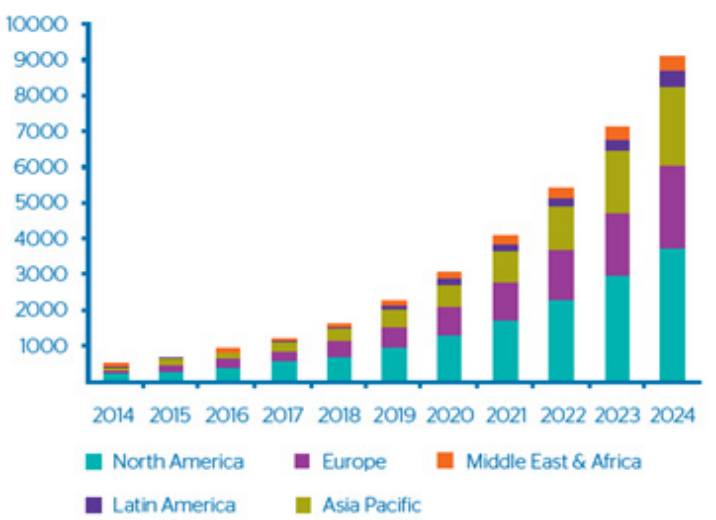

FabricsFigure (12): Market prediction for Smart Textiles 2014-2024

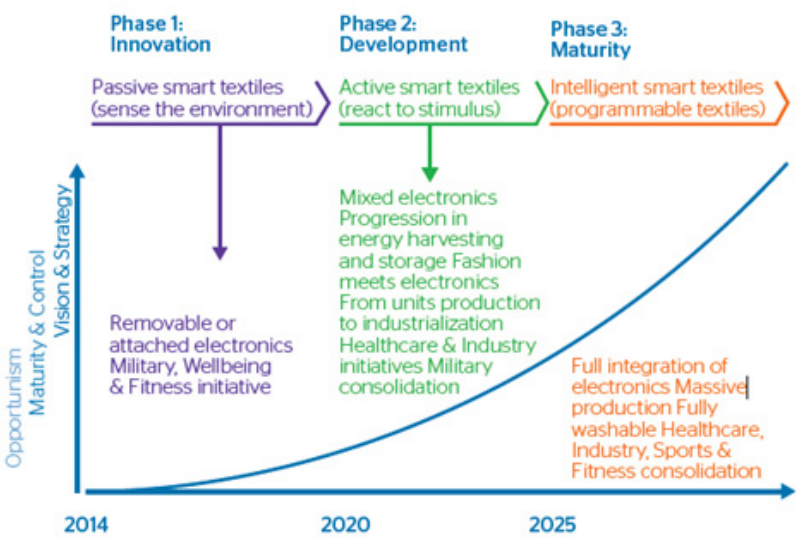

Figure (13): Evolution of Smart Fabrics according to the authors (L. Lancos, W.Thronickle, S. Ristol)(25)

\section{Methods of Applying for Possible Mass Produc- tions}

There are many different ways for applying new technological materials to produce smart textiles with an interactive ability starting with the first early stages of designing like, manufacturing the thread or the weaving stages to the later operations including dyeing, printing, and sewing. In this part of the paper we approach the printing process of smart textiles with an interactive ability, therefore, we discus examplesof applying process for mass productions and the most important stages during this process.

\subsection{PrintingThermochromic pigments:}

Whereas dying conveys a uniform colour, printing allows a range of different colours to be applied. Usually between 5 and 10 pastes are required for a single pattern. Colour may be supplied by either pigments or dyes. Printing is the process by which a localized coloration is made on the textile fabric. The printing is normally done by using dyes showing affinity to the fibre. On the other hand, it can be performed superficially with pigments, which could be fixed using thermocurable resins.

\subsubsection{Printing methods}

Direct printing: colour is applied directly to fabric in pattern \& location desired in finished fabric.

Screen printing: incredibly versatile, simple process separate mesh screens used for each colour .

Digital printing.

Ink-jet printing.

Electrostatic printing: prepared screen covered with powdered dye; passes through electric field \& pulled onto material.

\subsubsection{Printing Process}

Fabric preparation: The fabric should have uniform 
hydrophilicity and the surface should be free of fibres. Deposition of colorant on a dry textile surface: Operating in a continues way to deposit the colorant on the fabric surface.

Fixation of printed colour: It is possible by 3 ways physical, physico-chemical and chemical ways.

Elimination of thickener paste: Normally by means of washing.(26)

\subsection{Methods of Application of PhotochromicCol-} orants

\section{2. 1.Direct Coloration}

Billah et al. optimized the procedure for directcoloration of textile using photochromic colorants. According to the procedure, amount of colorants and auxiliaries were taken on the basis of fabric weight (2-3\% owf). First the aqueous dispersion of the colorant was prepared by milling the colorants with a dispersing agent and ceramic balls $(250 \mathrm{~g})$ in rotating honey jars for $18 \mathrm{~h}$. 1\% aqueous acetic acid was used to adjust the $\mathrm{pH}$ to 5.5 . The dyeing was started at $40 \mathrm{oC}$ temperature, increased to $60 \mathrm{oC}$ at $2 \mathrm{oC} / \mathrm{min}$ gradient and continued for $10 \mathrm{~min}$ at this temperature (Figure 11). Temperature was further raised to $90 \mathrm{oC}$ at $1 \mathrm{oC} / \mathrm{min}$ and the material run for one hour. Exhausted bath was drained after reducing the temperature to $40 \mathrm{oC}$ and the sample washed with cold water.

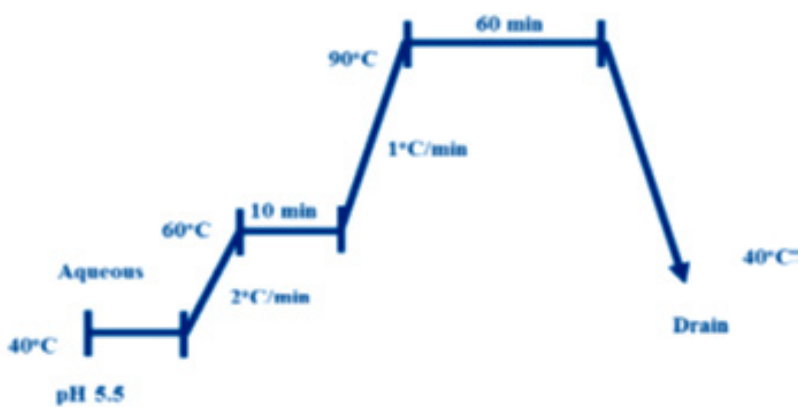

Figure(14). Schematic diagram of direct coloration method.

\subsubsection{Continuous Method}

For continuous application of photochromiccolorants on cotton fabric, first a small amount of dye is dissolved in acetone and stirred vigorously. The solution is then added drop wise to a binder formulation and mixed again vigorously. The dye solution is applied to the fabric using a padder under appropriate pressure. The fabric is dried at $80 \mathrm{oC}$ for 2 minutes in hot air, followed by curing at $140 \mathrm{oC}$ for 3 minutes again in a hot air oven or stenter.

\subsubsection{Screen Printing of Photochromic Colorants 6.2.3.1.As a Pigment}

Photochromic colorants can also be applied byscreen printing as a pigment. Anna et. al. hasdescribed the screen printing of photochromic colorants on textile fabric. To use photochromic colorants as a pigment, it is dissolved in acetone andsolution is added drop wise into a binder andthickener formulation. This formulation is referred to as printing paste of $0.05 \% \mathrm{w} / \mathrm{w}$, representing the concentration of the colorant in the print paste. Then using a plain mesh print screen, printing iscarried out, printed sample dried and cured at $140 \mathrm{oC}$ for $5 \mathrm{~min}$ in a hot air oven.

\subsubsection{As Disperse Dyes}

To use photochromic colorants as a disperse dye,first a colorant dispersion is prepared by mixing a photochromic colorant, an oil-based low foam wetting agent, a dispersing agent based on the disodium salt of a naphthalene sulphonic acidformaldehyde condensate and water and milling

for $30 \mathrm{~min}$ on a roller mill by using ceramic balls in a glass jar. A thickener solution is formulated using sodium alginate and water. The colorant dispersion is then mixed with the thickener solution to give a required colorant concentration in the printing paste. The fabric is printed, dried and cured at $140 \mathrm{oC}$ for five min. After fixation, reduction clearing is carried out, printed sample rinsed, washed with nonionic detergent solution $(1 \mathrm{~g} / \mathrm{l})$ for five min, rinsed again and dried in an oven at $60 \mathrm{oC} .(27)$

\section{Results and discussions}

- We conclude from our current study of Smart Printed Textiles and by recognizing its concept and its different patterns that these prints have the ability to "interact" with different factors and influences, whether external influences in the environment around the work or internal influences in the components of the infrastructure of the printed work itself. This "dynamic nature" differs in its artistic and physical characteristics, as well as in its aesthetic qualities from traditional prints which are known for their stability, which make it a necessitaty for an interactive textile printing designer to put a new work plan and a modern design methodology.

- We also conclude, through previous studies of a group of researchers and specialists in the field of designing smart textiles in general, including the design of interactive prints in particular, that it is not possible to develop specific steps for the designing process, due to a number of reasons mentioned in the capture of the following:

- The multiplicity of modern printing methods.

- The multiplicity ofthe printing materials and their diversity from each other in terms of chemical and physical properties.

- Differences in the desired functional or aesthetic objectives of the printed work.

- Therefore, a general methodological framework has 
been reached that can be followed by the smart textile printing designer, also a number of key factors and Design variables have been reached, which the designer can follow beside the appropriate scientific and technical steps to reach the desired interactive design concept.

- The researcher has also been able through the current study to reach the desired research objectives, which areidentifing the classification of smart printed textiles and patterns, as well as the most important materials and modern methods used in the field of application in order to reach the design and innovation of Smart Printed Textiles with interactive features.

\section{References}

(1) S. Baurley: "Interactive and experiential design in smart textile products and applications", Central Saint Martins College of Art and Design, The London Institute, Southampton Row, Woodhead Publishing Limited, London, p. 274 (2004)

(2) E. Heimdal and T. Lenau: "What is textile design research?' Submitted: Title of contribution:Physical tools for textile creativity and invention", DUCK - Journal for Research in Textiles and Textile Design, 22nd January, p.3, (2010)

(3) M. Mohamed, Technical Specifications of Materials, Vehicles and Smart Systems and their Applications in the Field of Industrial Design, Published Research, Journal of Science and Arts, Egypt, Volume 18, Issue 4, October, p. 31, (2006).

(4) L. Nilsson, A. Vallgrada and L. Worbin: "Designing With Smart Textiles: A New Research Programe”, Nordic Design Research Conference, Helsinki, p. 1, (2011)

(5) L. Worbin: "in the making - designing with smart textiles", The Nordic Textile Journal The Textile Research Centre, CTF, University of Borås, HB The Swedish School of Textiles, THS, p. 40, 19, 8:9, (2010)

(6) S.Morsümbül andE.P.AkçakocaKumbasar: "Photochromictextilematerials", Aegean International Textile and Advanced Engineering Conference (AITAE 2018(, journal citation and DOI., Published under licence by IOP Publishing Ltd, p. 3, (2018)

(7)A. Persson: "Knitted Circuits for Visual and TactileInteractive Expressions", Thesis for The Degree of Licentiat of Engineering,Department of Computer Science and Engineering, Chalmers University of Technology, Göteborg, Sweden, p. 29:30, (2009) (8) A. personne and L. Worbin: "Designing dynamic and irreversible textile patterns, using a non-chemical burn-out (ausbrenner) technique, Exploring Textiles As Materials For Interaction Design", The Nordic Textile Journal The Textile Research Centre, CTF, University of Borås, HB The Swedish School of Textiles, p. 67, 95:98, (2013)

(9) L. T H Berglin:'Interactive Textile Structures- Creating Multifunctional Textilesbased on Smart Materials", Thesis for The Degree of Doctor of Philosophy, Department of Computer Science and Engineering, Chalmers University of Technology, Goteborg, Sweden, p. 22:23, (2008)

(10) M. Ledendal: "Thermochromic textiles and sunlight activating systems: an alternative means to induce colour change", Submitted for the degree of Doctor of Philosophy, School of Textiles and Design, August,p. 36:37, 39, (2015)

(11) P. Kroes: "Design methodology and the nature of technical artefacts”, Design Studies, Vol 23, No. 3, Elsevier Science Ltd, Britin, p. 287-302, (2002)

(12) S. bonnemaison and C. macy: "responsive textile environments", canadian design research network, library and archives canada cataloguing in publication, canada, p. 28, (2007)

(13) Q. Wu and J. Hu: "Thermoelectric Textile Materials, Bringing Thermoelectricity into Reality", published by InTech, London, U.K., p. 23 \& 24, (2018)

(14) A. Willfahrt: "Screen Printed Thermoelectric Devices", Stuttgart Media University, Dept. Of Science and Technology Linköping University, Germany, April, p. 27, 28, (2014)

(15) M. Orrill and S. LeBlanc: "Printed thermoelectric materials and devices: Fabrication techniques, advantages, and challenges", Journal of Applied Polymer and Science, p. 1\& 6, (2016)

(16) M. Ferrara and M. Bengisu: "Intelligent design with chromogenic materials", Journal of the International Colour Association, p. $56: 62,(2014)$

(17) I. Verboven, J. Stryckers, V. Mecnika and others: "Printing Smart Designs of Light Emitting Devices with Maintained Textile Properties”, journal of Materials, p. 1, (2018)

(18) Dalal Abdullah Al-Sharif, Light Technology in Textiles as a Source of Design on Manikin, Ph.D. Thesis, College of Education for Home Economics, Umm Al-Qura University, Saudi Arabia, p. 77, (2009).

(19) R. Sharma and N. Bairagi: "The Role of Photoluminescent Pigments in Textiles", trends in textile engineering \& fashion technology, crimson puplisher, New York, USA, p. 2, (2018)

(20) S. Syduzzaman, S.U. Patwary, K. Farhanaz and S. Ahmed: "Smart Textiles and Nano -Technology: A General Overview", Textile Science \& Engineering, Vol. 5, Issue1, india, p. 3, 2, (2015) (21) N. Sarink,: "Characterization and Manufacturing of Textile Structures Based on Poly (vinylidene fluoride) Bi-component Fibres", Thesis for the Degree of Master in Science with a major in Textile Engineering The Swedish School of Textiles, university of Borås , p. 8 \& 9, (2014)

(22) N. Mohamed, Smart Clothing between the Data of Modern Technology and Design Requirements, Master Thesis, Faculty of Applied Arts, Helwan University, Egypt, p. 109, (2008).

(23) G. El-Gamal, Interactive Design of Ladies Printed Clothing Fabrics between Designer and Consumer, Journal of Science and Applied Arts, Damietta University, Egypt, Volume V, First Issue, January, pp. 164 and 166, (2018).

(24) L. Maleki, How to get changing patterns on a textile surface by using thermo chromic pigments and an inherently conductive Polymer, Degree of master in scienceinTextileTechnology, Bor॰s Textile School, Sweden, p. 15, (2010)

(25) L. Lancos, W.Thronickle, S. Ristol, Smart Fabrics - white papers, Thought Leadership, Atos Thought, December, p. 9, (2017). (26) J. M. Abdulkarim, A. K. Khsara, H. N. Al-Kalany, R. A. Alresly, "Impact of Properties of Thermochromic Pigments on Knitted Fabrics", International Journal of Scientific \& Engineering Research, Volume 7, Issue 4, April-P. 1694 \&1695, (2016).

(27) M. A. Chowdhury, M. Joshi and B. S. Butola, "Photochromic and Thermochromic Colorants in Textile Applications", Journal of Engineered Fibers and Fabrics, Volume 9, Issue 1, p. 111, (2014) 